\title{
Bilateral lumbar hernia
}

\author{
Iris Chung $^{1 \text { * }}$, MB, BS, MHKICBS, KY Wong ${ }^{2}$, FRCSEd, FHKAM (Surgery) \\ ${ }^{1}$ Department of Surgery, Queen Mary Hospital, Pokfulam, Hong Kong \\ ${ }^{2}$ Department of Surgery, Tung Wah Hospital, Sheung Wan, Hong Kong
}

Hong Kong Med J 2019;25:78-80

*Corresponding author: iris.intc@gmail.com

https://doi.org/10.12809/hkmj187410

Lumbar hernias were proposed in 1672 by Barbette but the first case was not published until 1731 wherein Garangeot reduced a lumbar hernia during autopsy'; the first repair was conducted 19 years later by Ravaton. ${ }^{2}$ The lumbar region, formed by the twelfth rib, iliac crest, erector spinae, and external oblique denotes the site for herniation. Lumbar hernias are most commonly categorised anatomically, as superior lumbar hernia (Grynfeltt-Lesshaft triangle), inferior lumbar hernia (Petit triangle), or diffuse involvement. Other classifications include aetiology (primary or secondary) and sac content (extra-, para- or intra-peritoneal); however, none of these categorisations have any treatment value. In 2007, Moreno-Egea et $\mathrm{al}^{2}$ proposed a preoperative classification with surgical implications (hernia size, location, content, aetiology, muscle atrophy and recurrence), but this has yet to be universally applied.

Owing to the rarity of lumbar hernia, a surgeon may only come across one case throughout their career. ${ }^{3}$ With only 300 cases reported, they comprise less than $2 \%$ of all abdominal hernias. ${ }^{3}$ Bilateral occurrences are even less frequently documented, with the first primary and secondary cases published in 2002 by Karmani et $\mathrm{al}^{4}$ and in 2006 by Bhasin et $\mathrm{al}^{5}$, respectively. Tung Wah Hospital performs an average of 500 hernia repairs annually, the majority of which are inguinal hernias; repair of lumbar hernias is uncommon, owing to their low incidence. Our most recent experience was in March 2017 when a 66-year-old man was referred to our centre for incidental finding of painless swellings over bilateral flanks which spontaneously reduced when the patient was supine; cough impulses were present. Aside from injuring his right lower ribs 2 months prior to presentation (treated conservatively), there was no trauma or surgical history. His past health includes hyperlipidaemia, benign prostatic hyperplasia, and obstructive sleep apnoea. His body mass index was $23 \mathrm{~kg} / \mathrm{m}^{2}$. A clinical diagnosis of bilateral reducible superior lumbar hernias (within the Grynfeltt-Lesshaft triangle) was made (Fig 1). Prior to consulting us, the patient underwent computed tomography imaging with findings compatible with our diagnosis. Open repair was performed under general anaesthesia with the patient lying prone. Dissection of the latissimus dorsi muscle via a linear incision revealed the hernias bounded superiorly by the twelfth rib, anteriorly by the posterior border of the internal oblique muscle, and posteriorly by the anterior border of the sacrospinalis muscle. Defects of $3 \mathrm{~cm}$ and $1.5 \mathrm{~cm}$ within the right and left superior lumbar triangle, respectively, were delineated; the sacs contained retroperitoneal fat and were easily reduced (Fig 2). Primary closure was

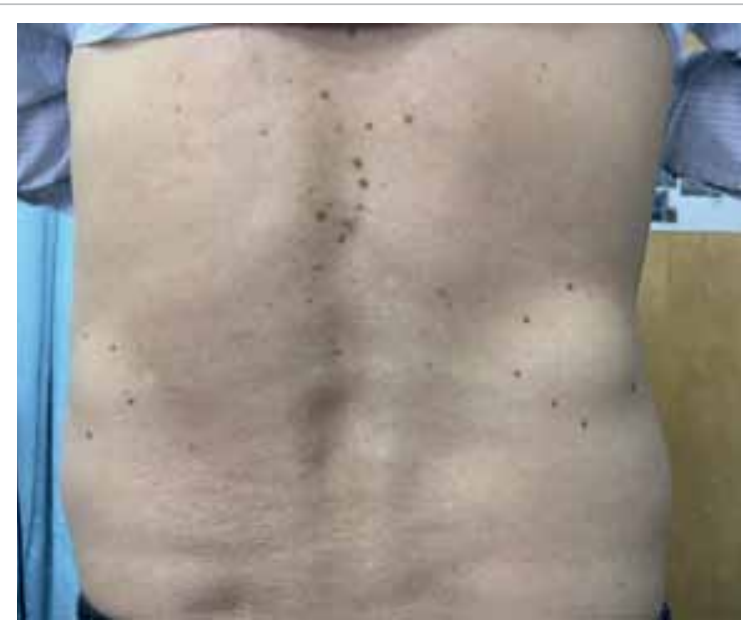

FIG I. Clinical photograph of the patient on the first presentation with bilateral flank swelling, more prominent on the right

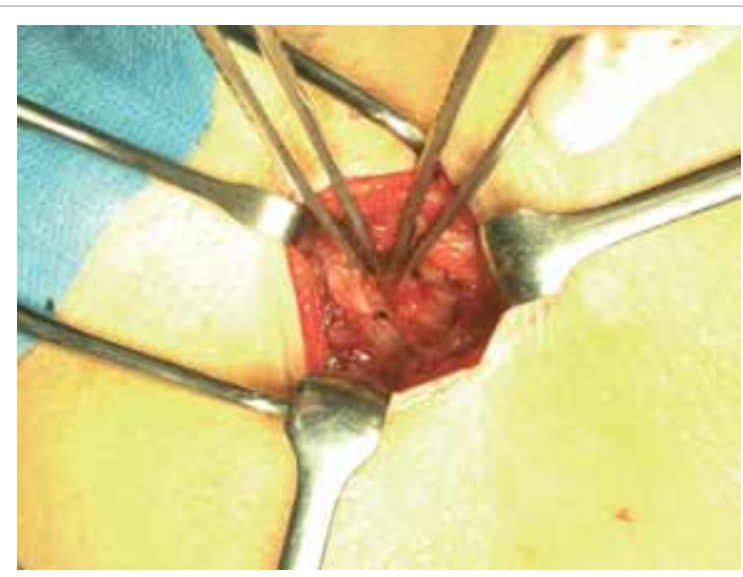

FIG 2. Intra-operative photograph showing the hernia sac freely dissected with protruding retroperitoneal fat contents successfully reduced 
performed with interrupted non-absorbable sutures, and an onlay polypropylene mesh anchored to the thoracolumbar fascia for reconstruction. No drain was inserted. Postoperative recovery was uneventful, and the patient was discharged from the hospital the following day. Interval follow-up reported no complications including wound infection, pain, or recurrence. Cosmetically, the patient was satisfied and remained asymptomatic on latest consultation.

Lumbar hernias are usually asymptomatic or present with non-specific complaints such as back or abdominal discomfort. A reducible mass with cough impulse may not always be present. Low suspicion can lead to misdiagnosis of alternative soft tissue pathologies like lipomas or retroperitoneal tumours. Sac contents can range from empty to intra- or retroperitoneal organs, which can produce atypical signs such as intestinal or urinary obstruction. Symptoms of back or abdominal pain with no obvious localising signs should suggest lumbar hernias as a differential, especially if there are risk factors. In all, $14 \%$ of cases have coexisting abdominal wall hernias; therefore, these patients should be screened. ${ }^{6}$ Imaging, such as computed tomography, can reveal any disrupted abdominal wall muscle layers, sac contents, and concomitant hernias. Although the physical findings were straightforward in our patient, the available imaging contributed towards diagnostic certainty, and we recommend the use of imaging to aid with preoperative planning.

In all, $20 \%$ of lumbar hernias are congenital, possibly from weakness in the abdominal muscle aponeurosis during development. ${ }^{7}$ The remaining $80 \%$ are acquired spontaneously (primary) or from preceding events (secondary). ${ }^{7}$ Associated risks include old age with weak abdominal muscles due to ageing; obesity and chronic respiratory conditions that increase intra-abdominal pressures; and extreme weight loss that decreases fat content, resulting in rupture of the twelfth neurovascular bundle orifice. Abdominal muscle weakening in secondary hernias can be due to trauma, infection, or postoperative complications from inadequate closure or subcostal nerve injury. Strangulation is rare as the neck is typically wide; however, reported incarceration rates are as high as $25 \%$, with $9 \%$ of acquired cases presenting acutely. ${ }^{1}$

Historically, the use of flaps was incorporated in lumbar hernia repair, as introduced by Dowd in $1907 .{ }^{2}$ It was not until the 1950 s to 60 s when Thorek ${ }^{8}$ and Hafner et $\mathrm{al}^{9}$ advocated the use of a mesh, and the 1990s when laparoscopic repairs were proposed by Burick and Parascandola. ${ }^{10}$ With limited cases to compare surgical approaches, the ideal method is inconclusive. Operative approaches largely depend on available facilities and the surgeons' expertise. Primary closure with interrupted tension-free sutures has been advocated for small defects, whereas larger hernias may be repaired using a non-absorbable mesh with or without anchoring to the twelfth rib or iliac crest. ${ }^{11}$ Mesh repairs have been suggested to reduce rates of recurrence, especially for patients in whom hernia occurrence is related to muscular atrophy or major deformities. ${ }^{5}$ In particular, sublay placement has been advocated for protecting the hernia orifice with help of underlying intra-abdominal pressure. Some centres suggest a double mesh technique whereby an onlay mesh is incorporated with a sublay mesh to ensure inclusion of the lower edge of the iliac crest, because this bony limit often impedes proper placement of mesh to fully cover the defect..$^{5,12}$ Laparoscopic repair with a sublay mesh via various transabdominal and extraperitoneal approaches have been explored but no meaningful comparisons have been made to conclude any definite advantages among laparoscopic approaches or in relation to open approaches.

Owing to their rarity, lumbar hernias are easily missed and misdiagnosed. High clinical suspicion is needed to avoid treatment delay. There is no recommendation for the ideal method of repair; therefore, surgical approaches should be tailored according to patient preference and surgeons' experience in managing this disease. If laparoscopic expertise is not available, open mesh repair is a safe alternative with satisfactory outcomes for small defects, as demonstrated in our patient.

\section{Author contributions}

All authors contributed to the concept and design of the study, acquisition of data, and interpretation of data, critical revision of the manuscript for important intellectual content. I Chung drafted the manuscript. All authors had full access to the data, contributed to the study, approved the final version for publication, and take responsibility for its accuracy and integrity.

\section{Conflicts of interest}

All authors have disclosed no conflicts of interest.

\section{Funding/support}

This research received no specific grant from any funding agency in the public, commercial, or not-for-profit sectors.

\section{Ethics approval}

Approval from an institutional review board or ethics committee was not required because our study did not involve clinical trials on human subjects. Any patient identifiers have been removed.

\section{References}

1. Petersen K, Snikeris J, Hall TS. Bleichner's hernia-lumbar hernia. Am J Case Rep 2013;14:26-9.

2. Moreno-Egea A, Baena EG, Calle MC, Martínez JA, Albasini JL. Controversies in the current management of lumbar hernias. Arch Surg 2007;142:82-8.

3. Ploneda-Valencia CF, Cordero-Estrada E, Castañeda- 
González LG, et al. Grynfelt-Lesshaft hernia a case report and review of the literature. Ann Med Surg (Lond) 2016;7:104-6.

4. Karmani S, Ember T, Davenport R. Congenital lumbar hernias: A case report. J Pediatr Surg 2002;37:921-2.

5. Bhasin SK, Khan AB, Sharma S. Bilateral petit's triangle hernia. JK Science 2006;8:163-4.

6. Fokou M, Fotso P, Ngowe Ngowe M, Essomba A, Sosso M. Strangulated or incarcerated spontaneous lumbar hernia as exceptional cause of intestinal obstruction: case report and review of the literature. World J Emerg Surg 2014;9:44.

7. Stamatiou D, Skandalakis JE, Skandalakis LJ, Mirilas P. Lumbar hernia: surgical anatomy, embryology, and technique of repair. Am Surg 2009;75:202-7.

8. Thorek M. Lumbar hernia. J Int Coll Surg 1950;14:367-93.

9. Hafner C, Wylie J Jr, Brush BE. Petit's lumbar hernia: repair with Marlex mesh. Arch Surg 1963;86:180-6.

10. Burick AJ, Parascandola S. Laparoscopic repair of a traumatic lumbar hernia: a case report. J Laparoendosc Surg 1996;6:259-62.

11. Esposito C, Settimi A, De Marco M, et al. Congenital lumbar hernia: two case reports and a review of the literature. J Paediatr Surg Spec 2009;3:40-2.

12. Bigolin AV, Rodrigues AP, Trevisan CG, et al. Petit lumbar hernia-a double-layer technique for tension-free repair. Int Surg 2014;99:556-9.

\section{Answers to CME Programme Hong Kong Medical Journal December 2018 issue}

Hong Kong Med J 2018;24:593-601

I. Group A Streptococcus disease in Hong Kong children: an overview
A
1. True
2. False
3. False
4. True
5. True
B
1. False
2. False
3. False
4. True
5. False

Hong Kong Med J 2018;24:617-22

II. Medication overuse headache: strategies for prevention and treatment using a multidisciplinary approach
A
1. False
2. True
3. False
4. False
5. True
B
1. True
2. False
3. False
4. True
5. True 